\title{
Synthesis of (E)-2-(arylbenzylidene)-2-((4-methoxyphenyl)amino) acetohydrazide derivatives and their antimicrobial activity
}

\author{
Basavapatna N. Prasanna Kumar ${ }^{* a}$, Kikkeri N. Mohana ${ }^{a}$, Lingappa Mallesha ${ }^{b}$ and \\ Nanjappagowda D. Rekha ${ }^{c}$
}

${ }^{a}$ Department of Studies in Chemistry, University of Mysore, Manasagangotri, Mysore 570 006, India

${ }^{b}$ PG Department of Chemistry, JSS College of Arts, Commerce and Science, Ooty Road, Mysore-25, India

${ }^{c} P G$ Department of Biotechnology, JSS College of Arts, Commerce and Science, Ooty Road, Mysore-25, India

CHRON I C LE

Article history:

Received March 27, 2013

Received in Revised form

July 27, 2013

Accepted 22 August 2013

Available online

24 August 2013

Keywords:

2,5-Disubstituted-1,3,4-oxadiazoles,

Iodobenzene diacetate

Oxidative cyclization

Antibacterial activity

Antifungal activity \begin{abstract}
A B S T R A C T
A series of some new (E)-2-(arylbenzylidene)-2-((4-methoxyphenyl)amino)acetohydrazides 4(a-j) have been conveniently synthesized by intermolecular oxidative cyclization of (E)-2(arylbenzylidene)-2-[(4-methoxyphenyl)amino]acetohydrazides promoted by iodobenzene diacetate as an oxidant. The structures of the synthesized compounds have been confirmed by ${ }^{1} \mathrm{H}$ NMR, IR, MS and elemental analysis. All the synthesized compounds were tested for their inhibitory action against clinically isolated strains i.e., Bacillus subtilis, Staphylococcus aureus, Xanthomonas campestris, Escherichia coli and Fusarium oxysporum. Compounds 4f (21-28 $\mathrm{mm}, 73.8 \%), \mathbf{4 i}(20-24 \mathrm{~mm}, 72.4 \%)$ and $\mathbf{4 j}(21-27 \mathrm{~mm}, 71.6 \%)$ demonstrated good antimicrobial activity against all the tested bacterial and fungal strains.
\end{abstract}

\section{Introduction}

In the past decades, the problem of multidrug resistant micro-organism has reached on alarming level around the world, and the synthesis of new anti-infective compounds has become an urgent need for the treatment of microbial infections. There are various problems arising with the use of antimicrobials such as local tissue irritation, interference with wound healing process, hypersensitivity reactions, system toxicity, narrow antimicrobial spectrum, and emergence of

* Corresponding author. Mob: $+91-90087-22226$

E-mail addresses: bnprasannak@gmail.com (B. N. Prasanna Kumar) 
resistance. $^{1,2}$ Therefore, the increasing clinical importance of drug resistant microbial pathogens has additional urgency in microbiological and antifungal research. Compounds containing heterocyclic ring systems continue to attract considerable interest due to the wide range of biological activities they possess. ${ }^{3}$ Among them five membered rings gained importace because of their versatile biological properties. ${ }^{3}$ In particular, compounds bearing 1,3,4-oxadiazoles nucleus are known to have unique angioedema and anti-inflammatory activities. ${ }^{4}$ Substituted oxadiazoles molecules possess other interesting properties such as analgesic ${ }^{5}$, antimicrobial ${ }^{6}$, antiviral ${ }^{7}$, anticonvulsant ${ }^{8}$, antihypertensive ${ }^{9}$, anti-proliferative ${ }^{10}$, enzyme Inhibitors ${ }^{11}, 5$-HT-receptor antagonists ${ }^{12}$ and inhibitors of muscle glycogen phosphorylase ${ }^{13}$.

Literature reveals that docking study of $N$-(hetarylmethyl)-aniline derivatives showed anticancer activity $^{14}$ and $N$-aryl- $N$-benzylamines were synthesized and evaluated for their antifungal activity, which was compared with their homoallylamine analogues that possessed an allyl group in the carbon next to the nitrogen atom. Results indicated that the absence of the allyl group caused an enhancement of the antifungal activity which could be correlated with the flexibility of the alkyl chain between both aromatic groups. ${ }^{15}$ This led to the preparation of $N$-substituted amines bearing a hetaryl fragments ( $N$-(hetarylmethyl)-anilines).

\section{Results and discussion}

\subsection{Chemistry}

The new compounds were prepared by using the synthetic strategy described in Scheme 1. The 2(4-methoxyphenylamino)acetohydrazide 2 was synthesized by the reaction of ethyl-(4methoxyphenyl)glycinate 1 with hydrazine hydrate in ethanol as per the reported procedure ${ }^{15}$.

The (E)-2-(arylbenzylidene)-2-((4-methoxyphenyl)amino)acetohydrazide (3a-j) was synthesized by the reaction of 2-(4-methoxyphenylamino)acetohydrazide 2 . It was refluxed with different substituted aldehydes in ethanolic solution for about $2 \mathrm{~h}$. A series of new 2,5-disubstituted-1,3,4oxadiazoles (Table 1) have been accomplished in excellent yields by the oxidation of $\mathbf{3 a - j}$ of various aryl aldehydes with one equivalent of iodobenzenediacetate (IBD) in dichloromethane. Compounds $\mathbf{3 a - j}$ and $\mathbf{4 a - j}$ were synthesized based on the reported procedure. ${ }^{16,17}$
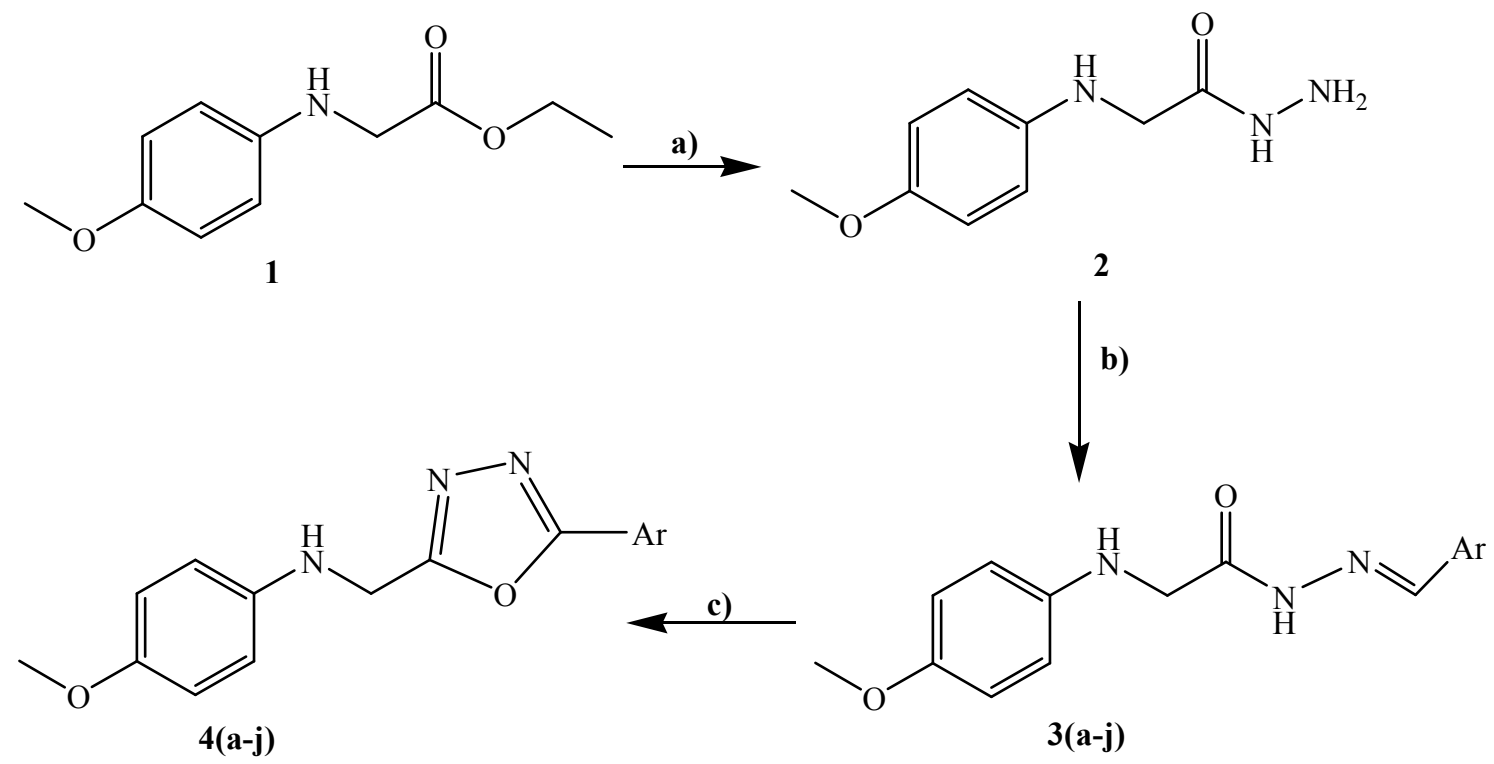

a) Hydrazine hydrate, ethanol, reflux 3 h, b) Aryl aldehydes, ethanol, reflux 2 h, c) IBD, DCM, reflux 2 h.

Scheme 1. Synthesis of compounds 4 
The IR spectra of the compound 3a exhibited characteristic bands at absorption bands at $1631 \mathrm{~cm}^{-1}$ and $3150 \mathrm{~cm}^{-1}$ due to for carbonyl and $\mathrm{NH}$ group, respectively. The ${ }^{1} \mathrm{H}$ NMR spectra of 3a showed two singlet due at $\mathrm{N}=\mathrm{CH}$ and $\mathrm{NH}$ at $\delta 8.58$ and $\delta 9.34$, respectively. The structure of all compounds 4a-j confirmed by their spectral (IR, LCMS, and ${ }^{1} \mathrm{H}$ NMR) and elemental analysis. The characterization of products $\mathbf{4 a - j}$ was based upon a careful comparison of their IR and ${ }^{1} \mathrm{H}$ NMR spectra with those of $\mathbf{3 a - j}$. IR spectra of $\mathbf{4 a}$ were found to be transparent in the region of $\mathrm{NH}$ stretch and $\mathrm{CO}$ stretch. In ${ }^{1} \mathrm{H}$ NMR spectra of $\mathbf{4 a}$ the disappearance of their singlet due to $\mathrm{N}=\mathrm{CH}$ around $\delta$ 8.4-8.6 and NH proton around $\delta$ 9.3-9.5, thus confirming the oxidation of $\mathbf{3 a - j}$ into $\mathbf{4 a - j}$. The mass

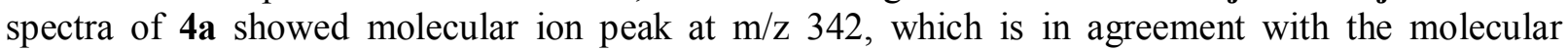
formula $\mathrm{C}_{18} \mathrm{H}_{19} \mathrm{~N}_{3} \mathrm{O}_{4}$.

Table 1. Chemical structure and physical data of 2,5-disubstituted-1,3,4-oxadiazoles (4a-j)<smiles>COc1ccc(NCc2nnc(-c3ccc(OC)cc3OCc3nnc(-c4c(F)cccc4Cl)o3)o2)cc1</smiles> 


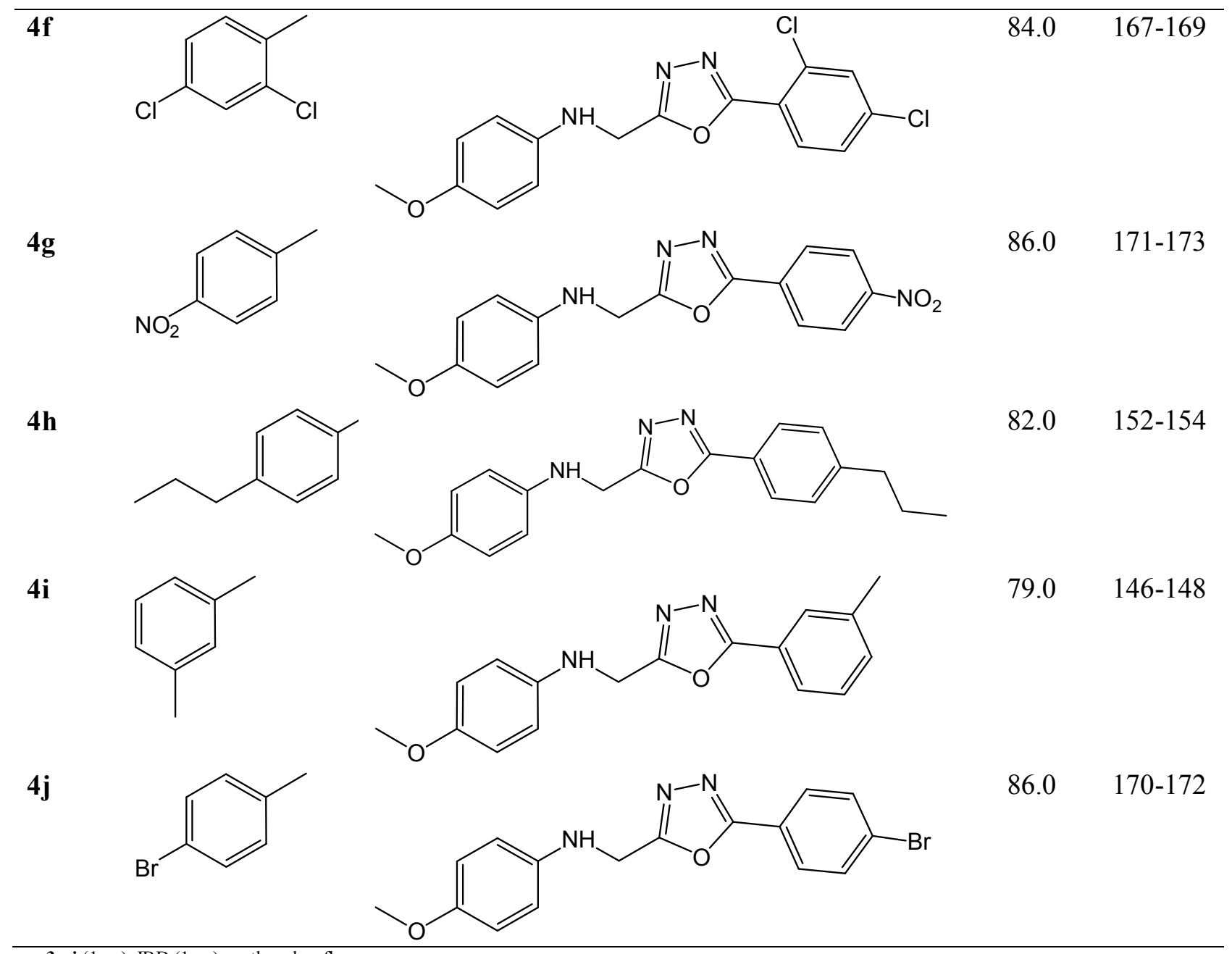

3a-j (1 eq), IBD (1 eq), methanol, reflux.

\subsection{Antimicrobial activity}

Compounds (4a-j) were tested in vitro for their antibacterial activity against two gram positive and two gram negative bacterial strains. Commercial antibiotics such as bacteriomycin and gentamycin were used as standard drugs. The results were compared with standard drugs and depicted in Table 2. Compound $\mathbf{4 f}$ was found to be more potent against gram positive and gram negative bacterial strains with the zone of inhibition respectively $21-28 \mathrm{~mm}$. Compounds $\mathbf{4 i}$ and $\mathbf{4 j}$ exhibited 20-24 mm and 21-27 mm, respectively against all the bacterial strains. Compounds $\mathbf{4 a}, \mathbf{4 b}$, $\mathbf{4 d}, \mathbf{4 e}$ and $\mathbf{4 g}$ were showed moderate antibacterial activity and compound $\mathbf{4 d}$ was found to be slightly active than $\mathbf{4 a}, \mathbf{4 e}, \mathbf{4 b}$ and $\mathbf{4 g}$. A compound $\mathbf{4 h}$ was weakly active against tested bacterial strains.

The in vitro antifungal activity of the new oxadiazole derivatives (4a-j) was studied against the fungal strain, Fusarium oxysporum. Nystatin was used as a standard drug and the results are given in Table 2. Compounds $\mathbf{4 f}$, $\mathbf{4 i}$ and $\mathbf{4 j}$ showed $73.8 \%, 72.4 \%$ and $71.6 \%$ inhibition, respectively when compared to standard drug (100\%). Compounds 4d (69.0\%), 4e (63.1\%), 4 b (56.4\%), 4 g (52.0 \%) and $4 \mathbf{a}(53.8 \%)$ exhibited moderate antifungal activity against tested fungal strain. On the other hand, the lowest antifungal effect was detected for compounds $\mathbf{4 h}(51.9 \%)$ and $\mathbf{4 c}(50.2 \%)$ against tested fungal strain. In the present study different groups attached to aryl ring as substituent linkage to 1,3,4oxadiazole ring. The close survey of antimicrobial efficacy indicated that the inhibition values of all the compounds exhibited a different range of activity against the tested strains. The biological results for compounds (4a-j) showed that the substitution pattern on phenyl ring appears to be vital for broad spectrum activity. 
Table 2. In vitro antibacterial and antifungal activities of the synthesized compounds

\begin{tabular}{|c|c|c|c|c|c|}
\hline \multirow[t]{2}{*}{ Compound } & \multicolumn{4}{|c|}{ Zone of inhibition in diameter $(\mathrm{mm})$} & $\%$ Inhibition \\
\hline & B. subtilis & S. aureus & X. campestris & E. coli & F. oxysporum \\
\hline $4 a$ & 18 & 18 & 17 & 16 & 53.8 \\
\hline $4 \mathrm{~b}$ & 18 & 19 & 19 & 22 & 56.4 \\
\hline $4 c$ & 12 & 13 & 12 & 13 & 50.2 \\
\hline $4 d$ & 21 & 20 & 19 & 23 & 69.0 \\
\hline $4 \mathrm{e}$ & 19 & 19 & 20 & 21 & 63.1 \\
\hline $4 f$ & 25 & 23 & 21 & 28 & 73.8 \\
\hline $4 \mathrm{~g}$ & 16 & 18 & 17 & 16 & 52.0 \\
\hline $4 \mathrm{~h}$ & 14 & 15 & 14 & 15 & 51.9 \\
\hline $4 \mathrm{i}$ & 22 & 21 & 20 & 24 & 72.4 \\
\hline $4 j$ & 23 & 22 & 21 & 27 & 71.6 \\
\hline Bacteriomycin & - & - & 34 & - & - \\
\hline Gentamycin & 35 & 30 & - & 35 & - \\
\hline Nystatin & - & - & - & - & 100 \\
\hline
\end{tabular}

\section{Conclusions}

In conclusion, a series of new 2,5-disubstituted-1,3,4-oxadiazoles (4a-j) were synthesized in good yield, characterized by different spectral studies and their antimicrobial activities have been evaluated. Compounds $\mathbf{4 f}, \mathbf{4 i}$ and $\mathbf{4 j}$ demonstrated good inhibition against microbial strains tested. The substituent on phenyl ring is responsible for the antimicrobial activity of these classes of agents. On the basis of their activity, these derivatives were identified as viable leads for further studies.

\section{Acknowledgments}

One of the authors (B. N. Prasanna Kumar) grateful to Synzene International Private Limited, Bangalore, India, given an opportunity to continue the higher education.

\section{Experimental}

\subsection{Materials and Methods}

All solvents and reagents were purchased from Merck Chemicals, India. Melting point was determined by VMP III apparatus. An elemental analysis was recorded on Vario MICRO superuser V1.3.2 Elementar. The IR spectra were recorded using $\mathrm{KBr}$ discs on IR Jasco 4100 infrared spectrophotometer. ${ }^{1} \mathrm{H}$ NMR spectra was recorded using $\mathrm{d}_{6}$-DMSO as solvent on Bruker DRX-500 spectrometer at $400 \mathrm{MHz}$. The mass spectrum was recorded using the instrument LC-MSD-Trap-XCT.

\subsection{Synthesis of 2-(4-methoxyphenylamino)acetohydrazide (2)}

A solution of ethyl-(4-methoxyphenyl)glycinate 1 (10 gm, $0.048 \mathrm{mmol})$ in ethanol was taken, hydrazine hydrate $(5 \mathrm{~mL})$ was added, and then reaction mixture refluxed for $3 \mathrm{~h}$. The reaction mixture was cooled and the solid formed was filtered and washed with chilled ethanol. Yield: $78 \%$. mp 116$118{ }^{\circ} \mathrm{C} .{ }^{1} \mathrm{H}$ NMR (DMSO-d $\left.6,400 \mathrm{MHz}\right) \delta: 4.51$ (s, 2H, NH $\mathrm{NH}_{2}$, 4.60 (s, 2H, -CH $), 7.10$ (dd, 2H, Ar$\mathrm{H}), 7.79$ (dd, 2H, Ar-H).

4.3. General procedure for the synthesis of (E)-2-(arylbenzylidene)-2-((4methoxyphenyl)amino)acetohydrazides (3a-j)

Compound 2 was refluxed with different aryl aldehydes in ethanolic solution $(10 \mathrm{~mL})$ for about 2 h. Reaction completion was confirmed by the thin layer chromatography (TLC). The reaction mass was cooled to 5 to $10{ }^{\circ} \mathrm{C}$ for $1 \mathrm{~h}$. The reaction mass was filtered and washed with ethanol. The obtained solid was dried to get the pure product. 


\subsection{1. (E)-N-(2,4-Dimethoxybenzylidene)-2-((4-methoxyphenyl)amino)acetohydrazide (3a)}

The compound 3a was obtained by the reaction of $2(1.0 \mathrm{gm}, 0.005 \mathrm{mmol})$ and 2,4dimethoxybenzaldehyde $(0.85 \mathrm{gm}, 0.005 \mathrm{mmol})$ using the general procedure described for $\mathbf{3}(\mathbf{a}-\mathbf{j})$. Yield $90 \%$ mp 151-153 ${ }^{\circ} \mathrm{C}$. IR $\left(\mathrm{KBr}, \mathrm{cm}^{-1}\right): 3300$ and $3150(\mathrm{~N}-\mathrm{H}), 3073(\mathrm{C}-\mathrm{H}), 1681(\mathrm{C}=\mathrm{N}), 1631$ $(\mathrm{C}=\mathrm{O}) .{ }^{1} \mathrm{H}$ NMR $\left(\mathrm{DMSO}_{6}, 400 \mathrm{MHz}\right) \delta: 3.87\left(\mathrm{~s}, 3 \mathrm{H},-\mathrm{OCH}_{3}\right), 3.88\left(\mathrm{~s}, 6 \mathrm{H},-\mathrm{OCH}_{3}\right), 4.60(\mathrm{~d}, 2 \mathrm{H}$, $\left.\mathrm{CH}_{2}\right), 6.35(\mathrm{~s}, \mathrm{NH}), 7.03(\mathrm{~d}, 2 \mathrm{H}, \mathrm{Ar}-\mathrm{H}), 7.44-7.48(\mathrm{~m}, 3 \mathrm{H}, \mathrm{Ar}-\mathrm{H}), 7.82(\mathrm{~d}, 2 \mathrm{H}, \operatorname{Ar}-\mathrm{H}), 8.58(\mathrm{~s}$, $\mathrm{N}=\mathrm{CH}), 9.34(\mathrm{~s}, \mathrm{NH})$.

\subsection{2. (E)-N-(2,3-Dimethoxybenzylidene)-2-((4-methoxyphenyl)amino)acetohydrazide (3b)}

The compound 3b was obtained by the reaction of $\mathbf{2}$ (1.0 gm, $0.005 \mathrm{mmol})$ and 2,3dimethoxybenzaldehyde $(0.85 \mathrm{gm}, 0.005 \mathrm{mmol})$ using the general procedure described for $\mathbf{3}(\mathbf{a}-\mathbf{j})$. Yield $84 \%$. mp 155-157 ${ }^{\circ} \mathrm{C}$. IR (KBr, cm $\left.{ }^{-1}\right): 3310$ and $3155(\mathrm{~N}-\mathrm{H}), 3070(\mathrm{C}-\mathrm{H}), 1680(\mathrm{C}=\mathrm{N}), 1634$ $(\mathrm{C}=\mathrm{O}),{ }^{1} \mathrm{H}$ NMR $\left(\mathrm{DMSO}_{6}, 400 \mathrm{MHz}\right) \delta: 3.87\left(\mathrm{~s}, 6 \mathrm{H},-\mathrm{OCH}_{3}\right), 3.88\left(\mathrm{~s}, 3 \mathrm{H},-\mathrm{OCH}_{3}\right), 4.59(\mathrm{~d}, 2 \mathrm{H},-$ $\left.\mathrm{CH}_{2}\right), 6.37$ (s, NH), 7.16-7.20 (m, 4H, Ar-H), 7.88-7.90 (m, 3H, Ar-H), 8.57 (s, N=CH), 9.28 (s, NH).

\subsection{3. (E)-N-(3-hydroxybenzylidene)-2-((4-methoxyphenyl)amino)acetohydrazide (3c)}

The compound $3 \mathbf{c}$ was obtained by the reaction of 2 (1.0 gm, $0.005 \mathrm{mmol})$ and 3hydroxybenzaldehyde $(0.63 \mathrm{gm}, 0.005 \mathrm{mmol})$ using the general procedure described for $\mathbf{3 ( a - j )})$. Yield $88 \%$. mp 160-162 ${ }^{\circ} \mathrm{C}$. IR (KBr, cm $\left.{ }^{-1}\right): 3314$ and $3171(\mathrm{~N}-\mathrm{H}), 3066(\mathrm{C}-\mathrm{H}), 1676(\mathrm{C}=\mathrm{N}), 1630(\mathrm{C}=\mathrm{O})$. ${ }^{1} \mathrm{H}$ NMR (DMSO-d 6 , $\left.400 \mathrm{MHz}\right) \delta: 3.87\left(\mathrm{~s}, 3 \mathrm{H},-\mathrm{OCH}_{3}\right), 4.58\left(\mathrm{~d}, 2 \mathrm{H},-\mathrm{CH}_{2}\right), 5.22(\mathrm{~s}, 1 \mathrm{H}, \mathrm{OH}), 6.38(\mathrm{~s}$, $\mathrm{NH}), 7.01(\mathrm{dd}, 2 \mathrm{H}, \mathrm{Ar}-\mathrm{H}), 7.23-7.36(\mathrm{~m}, 4 \mathrm{H}, \mathrm{Ar}-\mathrm{H}), 7.89(\mathrm{t}, 2 \mathrm{H}, \mathrm{Ar}-\mathrm{H}), 8.50(\mathrm{~s}, \mathrm{~N}=\mathrm{CH}), 9.20(\mathrm{~s}, \mathrm{NH})$.

\subsection{4. (E)-N-(3-methoxybenzylidene)-2-((4-methoxyphenyl)amino)acetohydrazide (3d)}

The compound 3d was obtained by the reaction of 2 (1.0 gm, $0.005 \mathrm{mmol})$ and 3methoxybenzaldehyde $(0.70 \mathrm{gm}, 0.005 \mathrm{mmol})$ using the general procedure described for $\mathbf{3 ( a - j})$. Yield $90 \%$. mp 171-173 ${ }^{\circ} \mathrm{C}$. IR (KBr, cm $\left.{ }^{-1}\right)$ : 3318 and $3170(\mathrm{~N}-\mathrm{H}), 3069(\mathrm{C}-\mathrm{H}), 1670(\mathrm{C}=\mathrm{N}), 1630(\mathrm{C}=\mathrm{O})$. ${ }^{1} \mathrm{H}$ NMR (DMSO-d $\left.6,400 \mathrm{MHz}\right) \delta: 3.87\left(\mathrm{~s}, 3 \mathrm{H},-\mathrm{OCH}_{3}\right), 3.88\left(\mathrm{~s}, 3 \mathrm{H},-\mathrm{OCH}_{3}\right), 4.59\left(\mathrm{~d}, 2 \mathrm{H},-\mathrm{CH}_{2}\right)$, 6.37 (s, NH), 7.04-7.10 (d, 4H, Ar-H), 7.59 (s, 1H, Ar-H), 7.66-7.75 (m, 3H, Ar-H). 8.51 (s, N=CH), $9.32(\mathrm{~s}, \mathrm{NH})$.

\subsection{5. (E)-N-(2-chloro-6-fluorobenzylidene)-2-((4-methoxyphenyl)amino)acetohydrazide (3e)}

The compound $3 \mathbf{e}$ was obtained by the reaction of 2 (1.0 gm, $0.005 \mathrm{mmol})$ and 2-chloro-6fluorobenzaldehyde $(0.81 \mathrm{gm}, 0.005 \mathrm{mmol})$ using the general procedure described for $\mathbf{3}(\mathbf{a}-\mathbf{j})$. Yield 84 \%. mp 149-151 ${ }^{\circ} \mathrm{C}$. IR (KBr, cm $\left.{ }^{-1}\right): 3306$ and $3170(\mathrm{~N}-\mathrm{H}), 3060(\mathrm{C}-\mathrm{H}), 1672(\mathrm{C}=\mathrm{N}), 1636(\mathrm{C}=\mathrm{O}) .{ }^{1} \mathrm{H}$ NMR (DMSO-d $6,400 \mathrm{MHz}) \delta: 3.88\left(\mathrm{~s}, 3 \mathrm{H},-\mathrm{OCH}_{3}\right), 4.59$ (d, 2H, $\left.-\mathrm{CH}_{2}\right), 6.38(\mathrm{~s}, \mathrm{NH}), 7.08(\mathrm{dd}, 2 \mathrm{H}$, Ar-H), 7.31-7.34 (t, 1H, Ar-H), 7.45-7.48 (m, 4H, Ar-H), 8.48 (s, N=CH), 9.29 (s, NH).

\subsection{6. (E)-N-(2,4-dichlorobenzylidene)-2-((4-methoxyphenyl)amino)acetohydrazide (3f)}

The compound $\mathbf{3 f}$ was obtained by the reaction of $2(1.0 \mathrm{gm}, 0.005 \mathrm{mmol})$ and 2,4dichlorobenzaldehyde $(0.90 \mathrm{gm}, 0.005 \mathrm{mmol})$ using the general procedure described for $\mathbf{3}(\mathbf{a}-\mathbf{j})$. Yield $90 \%$. mp 164-166 ${ }^{\circ} \mathrm{C}$. IR (KBr, cm $\left.{ }^{-1}\right)$ : 3308 and $3166(\mathrm{~N}-\mathrm{H}), 3069(\mathrm{C}-\mathrm{H}), 1676(\mathrm{C}=\mathrm{N}), 1630(\mathrm{C}=\mathrm{O})$. ${ }^{1} \mathrm{H}$ NMR (DMSO-d $\left.6,400 \mathrm{MHz}\right) \delta: 3.88\left(\mathrm{~s}, 6 \mathrm{H},-\mathrm{OCH}_{3}\right), 4.60\left(\mathrm{~d}, 2 \mathrm{H},-\mathrm{CH}_{2}\right), 6.37(\mathrm{~s}, \mathrm{NH}), 6.99-7.10$ (m, 8.26 Hz, 3H, Ar-H), 7.80-7.93 (m, 4H, Ar-H), 8.44 (s, N=CH), 9.33 (s, NH).

\subsection{7. (E)-2-((4-methoxyphenyl)amino)-N-(4-nitrobenzylidene)acetohydrazide (3g)}

The compound $\mathbf{3 g}$ was obtained by the reaction of $\mathbf{2}(1.0 \mathrm{gm}, 0.005 \mathrm{mmol})$ and 4nitrobenzaldehyde $(0.77 \mathrm{gm}, 0.005 \mathrm{mmol})$ using the general procedure described for $\mathbf{3}(\mathbf{a}-\mathbf{j})$. Yield 89 \%. mp 180-182 ${ }^{\circ} \mathrm{C}$. IR (KBr, cm $\left.{ }^{-1}\right): 3310$ and $3170(\mathrm{~N}-\mathrm{H}), 3074(\mathrm{C}-\mathrm{H}), 1680(\mathrm{C}=\mathrm{N}), 1635(\mathrm{C}=\mathrm{O}) .{ }^{1} \mathrm{H}$ 
NMR (DMSO-d $6,400 \mathrm{MHz}) \delta: 3.87\left(\mathrm{~s}, 3 \mathrm{H},-\mathrm{OCH}_{3}\right), 4.59$ (d, 2H, - $\left.\mathrm{CH}_{2}\right), 6.37$ (s, NH), 7.05 (dd, 2H, Ar-H), 7.66 (dd, 2H, Ar-H), 8.04-8.13 (m, 4H, Ar-H). 8.43 (s, N=CH), 9.31 (s, NH).

\subsection{8. (E)-N-(4-propylbenzylidene)-2-((4-methoxyphenyl)amino)acetohydrazide (3h)}

The compound $\mathbf{3 h}$ was obtained by the reaction of $\mathbf{2}(1.0 \mathrm{gm}, 0.005 \mathrm{mmol})$ and 4-

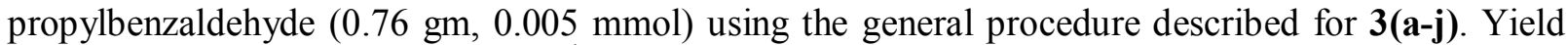
$90 \%$, mp 179-181 ${ }^{\circ} \mathrm{C}$. IR (KBr, cm $\left.{ }^{-1}\right): 3300$ and $3165(\mathrm{~N}-\mathrm{H}), 3070(\mathrm{C}-\mathrm{H}), 1678(\mathrm{C}=\mathrm{N}), 1634(\mathrm{C}=\mathrm{O})$. ${ }^{1} \mathrm{H}$ NMR (DMSO-d 6 , $\left.400 \mathrm{MHz}\right) \delta: 0.90\left(\mathrm{t}, 3 \mathrm{H}, \mathrm{CH}_{3}\right), 1.54-1.62\left(\mathrm{~m}, 2 \mathrm{H}, \mathrm{CH}_{2}\right), 2.48-2.50\left(\mathrm{t}, 2 \mathrm{H}, \mathrm{CH}_{2}\right)$, $3.87\left(\mathrm{~s}, 3 \mathrm{H},-\mathrm{OCH}_{3}\right), 4.59\left(\mathrm{~d}, 2 \mathrm{H},-\mathrm{CH}_{2}\right), 6.37(\mathrm{~s}, \mathrm{NH}), 7.08(\mathrm{dd}, 2 \mathrm{H}, \mathrm{Ar}-\mathrm{H}), 7.77-7.52(\mathrm{~m}, 4 \mathrm{H}, \mathrm{Ar}-$ $\mathrm{H}), 7.90(\mathrm{~d}, 2 \mathrm{H}, \mathrm{Ar}-\mathrm{H}) .8 .41(\mathrm{~s}, \mathrm{~N}=\mathrm{CH}), 9.36(\mathrm{~s}, \mathrm{NH})$.

\subsection{9. (E)-2-[(4-methoxyphenyl)amino]-N-((3-methylbenzylidene)acetohydrazide (3i)}

The compound $\mathbf{3 i}$ was obtained by the reaction of $2(1.0 \mathrm{gm}, 0.005 \mathrm{mmol})$ and 3methylbenzaldehyde $(0.62 \mathrm{gm}, 0.005 \mathrm{mmol})$ using the general procedure described for $\mathbf{3 ( a - j})$. Yield $85 \%$. mp 158-160 ${ }^{\circ} \mathrm{C}$. IR (KBr, cm $\left.{ }^{-1}\right): 3310$ and $3168(\mathrm{~N}-\mathrm{H}), 3075(\mathrm{C}-\mathrm{H}), 1671(\mathrm{C}=\mathrm{N}), 1632(\mathrm{C}=\mathrm{O}) .{ }^{1} \mathrm{H}$ NMR (DMSO-d $6,400 \mathrm{MHz}) \delta: 2.20\left(\mathrm{~s}, 3 \mathrm{H},-\mathrm{CH}_{3}\right), 3.88\left(\mathrm{~s}, 3 \mathrm{H},-\mathrm{OCH}_{3}\right), 4.60\left(\mathrm{~d}, 2 \mathrm{H},-\mathrm{CH}_{2}\right), 6.36(\mathrm{~s}, \mathrm{NH})$, 7.06 (dd, 2H, Ar-H), 7.77-7.84 (m, 5H, Ar-H), 8.10-8.15 (m, 1H, Ar-H). 8.41 (s, N=CH), 9.30 (s, NH).

\subsubsection{0. (E)-N-(4-bromobenzylidene)-2-[(4-methoxyphenyl)amino]acetohydrazide (3j)}

The compound $\mathbf{3} \mathbf{j}$ was obtained by the reaction of $\mathbf{2}(1.0 \mathrm{gm}, 0.005 \mathrm{mmol})$ and $4-$ bromolbenzaldehyde $(0.95 \mathrm{gm}, 0.005 \mathrm{mmol})$ using the general procedure described for $\mathbf{3}(\mathbf{a}-\mathbf{j})$. Yield $88 \%$. mp 177-179 ${ }^{\circ} \mathrm{C}$. IR (KBr, cm $\left.{ }^{-1}\right)$ : 3308 and $3168(\mathrm{~N}-\mathrm{H}), 3068(\mathrm{C}-\mathrm{H}), 1672(\mathrm{C}=\mathrm{N}), 1638(\mathrm{C}=\mathrm{O})$. ${ }^{1} \mathrm{H}$ NMR (DMSO-d 6 , $\left.400 \mathrm{MHz}\right) \delta: 3.88\left(\mathrm{~s}, 3 \mathrm{H},-\mathrm{OCH}_{3}\right), 4.60\left(\mathrm{~d}, 2 \mathrm{H},-\mathrm{CH}_{2}\right), 6.36(\mathrm{~s}, \mathrm{NH}), 7.06-7.18$ (m, 4H, Ar-H), 7.46 (d, 2H, Ar-H), 8.1 (d, 2H, Ar-H), 8.47 (s, N=CH), 9.32 (s, NH).

\subsection{General procedure for the synthesis of 2,5-disubstituted-1,3,4-oxadiazoles (4a-j)}

(E)-2-(arylbenzylidene)-2-((4-methoxyphenyl)amino)acetohydrazides (3a-j) was dissolved in dichloromethane and IBD were added to it. The contents were stirred for $2 \mathrm{~h}$ and the progress of the reaction was monitored by TLC. The solvent was removed and the residue was taken in petroleum ether and stirred for $30 \mathrm{~min}$. The solid thus obtained was filtered, washed with petroleum ether and dried to afford $(\mathbf{4 a - j})$.

\subsubsection{N-((5-(2,4-Dimethoxyphenyl)-1,3,4-oxadiazole-2-yl)methyl)-4-methoxyaniline (4a)}

The compound 4a was obtained by the reaction of 3a (1.0 gm, $0.003 \mathrm{mmol})$ and iodobenzene diacetate (1.12 gm, $0.0034 \mathrm{mmol})$ using the general procedure described for $\mathbf{4}(\mathbf{a}-\mathbf{j})$. White solid. FTIR $\left(\mathrm{KBr}, \mathrm{cm}^{-1}\right): 3060$ (aromatic C-H), $2929\left(\mathrm{C}-\mathrm{H}\right.$ of $\left.\mathrm{CH}_{2}\right), 1680(\mathrm{C}=\mathrm{N}), 1464(\mathrm{C}=\mathrm{C}), 1375(\mathrm{C}-\mathrm{N})$, 1255 (C-O). ${ }^{1} \mathrm{H}$ NMR (DMSO-d $\left.6,400 \mathrm{MHz}\right) \delta: 3.88$ (s, 3H, -OCH $), 3.89$ (s, 6H, - $\left.\mathrm{OCH}_{3}\right), 4.59$ (d, 2H, $\left.-\mathrm{CH}_{2}\right), 6.34(\mathrm{~s}, 1 \mathrm{H}, \mathrm{NH}), 7.09(\mathrm{~d}, 2 \mathrm{H}, \mathrm{Ar}-\mathrm{H}), 7.50-7.58(\mathrm{~m}, 3 \mathrm{H}, \mathrm{Ar}-\mathrm{H}), 7.92$ (d, 2H, Ar-H). MS (ESI) $\mathrm{m} / z$ : 342 (Expected mass: 341). Anal. Calcd. for $\mathrm{C}_{18} \mathrm{H}_{19} \mathrm{~N}_{3} \mathrm{O}_{4}$ ( in \%): $\mathrm{C}, 63.33$; H, 5.61; N, 12.31. Found: C, 63.25; H, 5.60; N, 12.25 .

\subsubsection{N-((5-(2,3-Dimethoxyphenyl)-1,3,4-oxadiazole-2-yl)methyl)-4-methoxyaniline (4b)}

The compound $\mathbf{4 b}$ was obtained by the reaction of $\mathbf{3 b}(1.0 \mathrm{gm}, 0.003 \mathrm{mmol})$ and iodobenzene diacetate $(1.12 \mathrm{gm}, 0.0034 \mathrm{mmol})$ using the general procedure described for $\mathbf{4}(\mathbf{a}-\mathbf{j})$. White solid. FTIR $\left(\mathrm{KBr}, \mathrm{cm}^{-1}\right): 3070$ (aromatic C-H), $2936\left(\mathrm{C}-\mathrm{H}\right.$ of $\left.\mathrm{CH}_{2}\right), 1672(\mathrm{C}=\mathrm{N}), 1470(\mathrm{C}=\mathrm{C}), 1384(\mathrm{C}-\mathrm{N})$, $1243(\mathrm{C}-\mathrm{O}) .{ }^{1} \mathrm{H}$ NMR (DMSO-d $\left.6,400 \mathrm{MHz}\right) \delta: 3.88\left(\mathrm{~s}, 6 \mathrm{H},-\mathrm{OCH}_{3}\right), 3.89$ (s, 3H, $\left.-\mathrm{OCH}_{3}\right), 4.59$ (d, $\left.2 \mathrm{H},-\mathrm{CH}_{2}\right), 6.38$ (s, 1H, NH), 7.08 (d, 2H, Ar-H), 7.16-7.20 (d, 2H, Ar-H), 7.85-7.88 (m, 3H, Ar-H). MS (ESI) $m / z$ : 342 (Expected mass: 341). Anal. Calcd. for $\mathrm{C}_{18} \mathrm{H}_{19} \mathrm{~N}_{3} \mathrm{O}_{4}$ ( in \%): C, 63.33; H, 5.61; N, 12.31. Found: C, 63.22; H, 5.58; N, 12.25 . 


\subsubsection{3-(5-((4-Methoxyphenyl)amino)methyl)-1,3,4-oxadiazole-2-yl)phenol (4c)}

The compound $4 \mathbf{c}$ was obtained by the reaction of $3 \mathbf{c}(1.0 \mathrm{gm}, 0.0033 \mathrm{mmol})$ and iodobenzene diacetate (1.29 gm, $0.004 \mathrm{mmol})$ using the general procedure described for 4(a-j). Brown solid. FTIR $\left(\mathrm{KBr}, \mathrm{cm}^{-1}\right): 3515(\mathrm{OH}), 3068$ (aromatic C-H), $2930\left(\mathrm{C}-\mathrm{H}\right.$ of $\left.\mathrm{CH}_{2}\right), 1652(\mathrm{C}=\mathrm{N}), 1469(\mathrm{C}=\mathrm{C})$, $1388(\mathrm{C}-\mathrm{N}), 1250(\mathrm{C}-\mathrm{O}) .{ }^{1} \mathrm{H}$ NMR (DMSO-d $\left.6,400 \mathrm{MHz}\right) \delta: 3.86\left(\mathrm{~s}, 3 \mathrm{H},-\mathrm{OCH}_{3}\right), 4.60\left(\mathrm{~d}, 2 \mathrm{H},-\mathrm{CH}_{2}\right)$, $5.20(\mathrm{~s}, 1 \mathrm{H}, \mathrm{OH}), 6.35(\mathrm{~s}, 1 \mathrm{H}, \mathrm{NH}), 6.88(\mathrm{dd}, 2 \mathrm{H}, \mathrm{Ar}-\mathrm{H}), 7.22-7.31(\mathrm{~m}, 2 \mathrm{H}, \mathrm{Ar}-\mathrm{H}), 7.55(\mathrm{~s}, 1 \mathrm{H}, \mathrm{Ar}-$ H), 7.88 (d, 2H, Ar-H). MS (ESI) m/z: 298 (Expected mass: 297). Anal. Calcd. for $\mathrm{C}_{16} \mathrm{H}_{15} \mathrm{~N}_{3} \mathrm{O}_{3}$ (in \%): C, 64.64; H, 5.09; N, 14.13. Found: C, 64.42; H, 4.89; N, 14.10.

\subsubsection{4-Methoxy-N-((5-(3-methoxyphenyl)-1,3,4-oxadiazole-2-yl)methyl]aniline (4d)}

The compound $\mathbf{4 d}$ was obtained by the reaction of $\mathbf{3 d}(1.0 \mathrm{gm}, 0.0032 \mathrm{mmol})$ and iodobenzene diacetate (1.23 gm, $0.0038 \mathrm{mmol})$ using the general procedure described for $\mathbf{4}(\mathbf{a}-\mathbf{j})$. White solid. FTIR $\left(\mathrm{KBr}, \mathrm{cm}^{-1}\right): 3060$ (aromatic C-H), $2944\left(\mathrm{C}-\mathrm{H}\right.$ of $\left.\mathrm{CH}_{2}\right), 1628(\mathrm{C}=\mathrm{N}), 1430(\mathrm{C}=\mathrm{C}), 1358(\mathrm{C}-\mathrm{N})$, 1247 (C-O). ${ }^{1} \mathrm{H}$ NMR (DMSO-d $\left.6,400 \mathrm{MHz}\right) \delta: 3.88$ (s, 6H, - $\left.\mathrm{OCH}_{3}\right), 4.58\left(\mathrm{~d}, 2 \mathrm{H},-\mathrm{CH}_{2}\right), 6.35(\mathrm{~s}, 1 \mathrm{H}$, NH), 7.07 (d, 2H, Ar-H), 7.59-7.66 (m, 4H, Ar-H), 7.70 (s, 1H, Ar-H). MS (ESI) m/z: 313 (Expected mass: 311). Anal. Calcd. for $\mathrm{C}_{17} \mathrm{H}_{17} \mathrm{~N}_{3} \mathrm{O}_{3}$ ( in \%): C, 65.88; H, 5.50; N, 13.50. Found: $\mathrm{C}, 60.50 ; \mathrm{H}$, $4.64 ; \mathrm{N}, 13.55$.

\subsubsection{N-((5-(2-Chloro-6-fluorophenyl)-1,3,4-oxadiazole-2-yl)methyl)-4-methoxyaniline (4e)}

The compound $4 \mathbf{e}$ was obtained by the reaction of $3 \mathbf{e}(1.0 \mathrm{gm}, 0.003 \mathrm{mmol})$ and iodobenzene diacetate (1.15 gm, $0.0035 \mathrm{mmol})$ using the general procedure described for $\mathbf{4}(\mathbf{a}-\mathbf{j})$. Pale brown solid. FT-IR (KBr, cm $\left.{ }^{-1}\right): 3072$ (aromatic C-H), $2961\left(\mathrm{C}-\mathrm{H}\right.$ of $\left.\mathrm{CH}_{2}\right), 1660(\mathrm{C}=\mathrm{N}), 1486(\mathrm{C}=\mathrm{C}), 1380(\mathrm{C}-\mathrm{N})$, 1236 (C-O). ${ }^{1} \mathrm{H}$ NMR (DMSO-d $\left.6,400 \mathrm{MHz}\right) \delta: 3.89$ (s, 3H, - $\left.\mathrm{OCH}_{3}\right), 4.59$ (d, 2H, - $\left.\mathrm{CH}_{2}\right), 6.36(\mathrm{~s}, 1 \mathrm{H}$, $\mathrm{NH}), 7.06$ (dd, 2H, Ar-H), 7.10-7.13 (t, 1H, Ar-H), 7.40-7.49 (m, 2H, Ar-H), 7.87 (dd, 2H, Ar-H), MS (ESI) $m / z: 335$ (Expected mass: 333). Anal. Calcd. for $\mathrm{C}_{16} \mathrm{H}_{13} \mathrm{ClFN}_{3} \mathrm{O}_{2}$ ( in \%): C, 57.58; $\mathrm{H}, 3.93$; $\mathrm{N}, 12.59$. Found: $\mathrm{C}, 57.65 ; \mathrm{H}, 3.99 ; \mathrm{N}, 12.50$.

\subsubsection{N-((5-(2,4-Dichlorophenyl)-1,3,4-oxadiazole-2-yl)methyl)-4-methoxyaniline (4f)}

The compound $\mathbf{4 f}$ was obtained by the reaction of $\mathbf{3 f}(1.0 \mathrm{gm}, 0.0028 \mathrm{mmol})$ and iodobenzene diacetate $(1.1 \mathrm{gm}, 0.0031 \mathrm{mmol})$ using the general procedure described for $\mathbf{4}(\mathbf{a}-\mathbf{j})$. White solid. FT-IR $\left(\mathrm{KBr}, \mathrm{cm}^{-1}\right)$ : 3070 (aromatic C-H), $2944\left(\mathrm{C}-\mathrm{H}\right.$ of $\left.\mathrm{CH}_{2}\right), 1629(\mathrm{C}=\mathrm{N}), 1480(\mathrm{C}=\mathrm{C}), 1386(\mathrm{C}-\mathrm{N}), 1245$ (C-O). ${ }^{1} \mathrm{H}$ NMR (DMSO-d $\left.6,400 \mathrm{MHz}\right) \delta: 3.88\left(\mathrm{~s}, 3 \mathrm{H},-\mathrm{OCH}_{3}\right), 4.60\left(\mathrm{~d}, 2 \mathrm{H},-\mathrm{CH}_{2}\right), 6.35(\mathrm{~s}, 1 \mathrm{H}, \mathrm{NH})$, 7.01 (dd, 2H, Ar-H), 7.76 (d, 2H, Ar-H), 7.88-7.94 (m, 3H, Ar-H). MS (ESI) m/z: 352 (Expected mass: 349). Anal. Calcd. for $\mathrm{C}_{15} \mathrm{H}_{10} \mathrm{Cl}_{2} \mathrm{~N}_{3} \mathrm{O}_{3}$ ( in \%): C, 53.75; $\mathrm{H}, 3.01 ; \mathrm{N}, 12.54$. Found: $\mathrm{C}, 53.58 ; \mathrm{H}$, $3.05 ; \mathrm{N}, 12.34$.

\subsubsection{N-((5-(4-Nitrophenyl)-1,3,4-oxadiazole-2-yl)methyl)-4-methoxyaniline (4g)}

The compound $\mathbf{4 g}$ was obtained by the reaction of $\mathbf{3 g}(1.0 \mathrm{gm}, 0.003 \mathrm{mmol})$ and iodobenzene diacetate $(1.18 \mathrm{gm}, 0.0036 \mathrm{mmol})$ using the general procedure described for $\mathbf{4}(\mathbf{a}-\mathbf{j})$. Yellow solid. FT-IR (KBr, cm $\left.{ }^{-1}\right): 3065$ (aromatic C-H), $2950\left(\mathrm{C}-\mathrm{H}\right.$ of $\left.\mathrm{CH}_{2}\right), 1634(\mathrm{C}=\mathrm{N}), 1481(\mathrm{C}=\mathrm{C}), 1380(\mathrm{C}-\mathrm{N})$, 1241 (C-O). ${ }^{1} \mathrm{H}$ NMR (DMSO-d $\left.6,400 \mathrm{MHz}\right) \delta: 3.87$ (s, 3H, - $\left.\mathrm{OCH}_{3}\right), 4.58\left(\mathrm{~d}, 2 \mathrm{H},-\mathrm{CH}_{2}\right), 6.34(\mathrm{~s}, 1 \mathrm{H}$, NH), 7.10-7.18 (m, 4H, Ar-H), 7.76 (d, 2H, Ar-H), 8.10 (d, 2H, Ar-H). MS (ESI) m/z: 327 (Expected mass: 326). Anal. Calcd. for $\mathrm{C}_{16} \mathrm{H}_{14} \mathrm{~N}_{4} \mathrm{O}_{4}$ ( in \%): $\mathrm{C}, 58.89 ; \mathrm{H}, 4.32 ; \mathrm{N}, 17.17$. Found: $\mathrm{C}, 58.80 ; \mathrm{H}$, $4.30 ; \mathrm{N}, 17.12$.

\subsubsection{4-Methoxy-N-((5-(4-propylphenyl)-1,3,4-oxadiazole-2-yl)methyl)aniline (4h)}

The compound $\mathbf{4 h}$ was obtained by the reaction of $\mathbf{3 h}(1.0 \mathrm{gm}, 0.003 \mathrm{mmol})$ and iodobenzene diacetate $(1.15 \mathrm{gm}, 0.0035 \mathrm{mmol})$ using the general procedure described for $\mathbf{4}(\mathbf{a}-\mathbf{j})$. White solid. FTIR $\left(\mathrm{KBr}, \mathrm{cm}^{-1}\right)$ : 3075 (aromatic $\left.\mathrm{C}-\mathrm{H}\right), 2950\left(\mathrm{C}-\mathrm{H}\right.$ of $\left.\mathrm{CH}_{2}\right), 1670(\mathrm{C}=\mathrm{N}), 1462(\mathrm{C}=\mathrm{C}), 1382(\mathrm{C}-\mathrm{N})$, 
1229 (C-O). ${ }^{1} \mathrm{H}$ NMR (DMSO-d $\left.6,400 \mathrm{MHz}\right) \delta: 0.92\left(\mathrm{t}, 3 \mathrm{H}, \mathrm{CH}_{3}\right), 1.54-1.62\left(\mathrm{~m}, 2 \mathrm{H}, \mathrm{CH}_{2}\right), 2.49-2.51$

(t, 2H, $\left.\mathrm{CH}_{2}\right), 3.87\left(\mathrm{~s}, 3 \mathrm{H},-\mathrm{OCH}_{3}\right), 4.59\left(\mathrm{~d}, 2 \mathrm{H},-\mathrm{CH}_{2}\right), 6.35(\mathrm{~s}, 1 \mathrm{H}, \mathrm{NH}), 7.10(\mathrm{dd}, 2 \mathrm{H}, \mathrm{Ar}-\mathrm{H}), 7.42$ (dd, 2H, Ar-H), 7.81 (d, 2H, Ar-H), 7.89-7.92 (m, 2H, Ar-H). MS (ESI) m/z: 324 (Expected mass: 333). Anal. Calcd. for $\mathrm{C}_{19} \mathrm{H}_{21} \mathrm{~N}_{3} \mathrm{O}_{2}$ ( in \%): C, 70.57; H, 6.55; N, 12.99. Found: C, 70.62; H, 6.52; N, 12.95 .

\subsubsection{4-Methoxy-N-((5-(m-tolyl)-1,3,4-oxadiazole-2-yl)methyl)aniline (4i)}

The compound $4 \mathbf{i}$ was obtained by the reaction of $3 \mathbf{i}(1.0 \mathrm{gm}, 0.0033 \mathrm{mmol})$ and iodobenzene diacetate $(1.3 \mathrm{gm}, 0.004 \mathrm{mmol})$ using the general procedure described for $\mathbf{4}(\mathbf{a}-\mathbf{j})$. White solid. FT-IR $\left(\mathrm{KBr}, \mathrm{cm}^{-1}\right): 3063$ (aromatic C-H), $2934\left(\mathrm{C}-\mathrm{H}\right.$ of $\left.\mathrm{CH}_{2}\right), 1688(\mathrm{C}=\mathrm{N}), 1469(\mathrm{C}=\mathrm{C}), 1385(\mathrm{C}-\mathrm{N}), 1231$ (C-O). ${ }^{1} \mathrm{H}$ NMR (DMSO-d $\left.6,400 \mathrm{MHz}\right) \delta: 2.22\left(\mathrm{~s}, 3 \mathrm{H},-\mathrm{CH}_{3}\right), 3.88\left(\mathrm{~s}, 3 \mathrm{H},-\mathrm{OCH}_{3}\right), 4.59(\mathrm{~d}, 2 \mathrm{H},-$ $\mathrm{CH}_{2}$ ), 6.35 (s, 1H, NH), 7.17 (dd, 2H, Ar-H), 7.51 (s, 1H, Ar-H), 7.77-7.82 (m, 4H, Ar-H). MS (ESI) m/z: 297 (Expected mass: 295). Anal. Calcd. for $\mathrm{C}_{17} \mathrm{H}_{17} \mathrm{~N}_{3} \mathrm{O}_{2}$ ( in \%): C, 69.14; H, 5.80; N, 14.23. Found: C, 69.10; H, 5.75; N, 14.18 .

\subsubsection{0. $\mathrm{N}$-[\{5-(4-Bromophenyl)-1,3,4-oxadiazole-2-yl\}methyl]-4-methoxyaniline (4j)}

The compound $\mathbf{4} \mathbf{j}$ was obtained by the reaction of $\mathbf{3 j}(1.0 \mathrm{gm}, 0.0027 \mathrm{mmol})$ and iodobenzene diacetate (1.34 gm, $0.004 \mathrm{mmol})$ using the general procedure described for $\mathbf{4}(\mathbf{a}-\mathbf{j})$. White solid. FT-IR $\left(\mathrm{KBr}, \mathrm{cm}^{-1}\right)$ : 3055 (aromatic C-H), $2921\left(\mathrm{C}-\mathrm{H}\right.$ of $\left.\mathrm{CH}_{2}\right), 1682(\mathrm{C}=\mathrm{N}), 1465(\mathrm{C}=\mathrm{C}), 1380(\mathrm{C}-\mathrm{N}), 1240$ (C-O). ${ }^{1} \mathrm{H}$ NMR (DMSO-d $\left.6,400 \mathrm{MHz}\right) \delta: 3.87$ (s, 3H, $\left.-\mathrm{OCH}_{3}\right), 4.60\left(\mathrm{~d}, 2 \mathrm{H},-\mathrm{CH}_{2}\right), 6.34(\mathrm{~s}, 1 \mathrm{H}, \mathrm{NH})$, 7.07 (d, 2H, Ar-H), 7.40 (d, 2H, Ar-H), 7.77 (d, 2H, Ar-H), 7.95 (d, 2H, Ar-H). MS (ESI) m/z: 362 (Expected mass: 360). Anal. Calcd. for $\mathrm{C}_{16} \mathrm{H}_{14} \mathrm{BrN}_{3} \mathrm{O}_{2}$ (in \%): $\mathrm{C}, 53.55 ; \mathrm{H}, 3.92 ; \mathrm{N}, 11.67$. Found: $\mathrm{C}$, $53.23 ; \mathrm{H}, 3.81 ; \mathrm{N}, 11.7$.

\subsection{Antibacterial activity}

Antibacterial activity of the newly synthesized compounds was determined in DMF by disc diffusion method on nutrient agar medium ${ }^{18}$ by using Gram-positive bacteria (Bacillus subtilis and Staphylococcus aureus) and Gram-negative bacteria (Xanthomonas campestris and Escherichia coli). In each Petri plate the sterile media (Nutrient Agar Medium, $15 \mathrm{~mL}$ ) was uniformly smeared with cultures of both bacteria. Sterile discs of $10 \mathrm{~mm}$ diameter (Hi-Media) was placed in the Petri plates, to which $50 \mu \mathrm{L}(1 \mathrm{mg} / \mathrm{ml}$ i.e., $50 \mu \mathrm{g} /$ disc $)$ of the new compounds were added. This included $50 \mu \mathrm{L}$ of DMF as negative, bacteriomycin and gentamycin as positive control. The plates were incubated at 37 $\pm 2{ }^{\circ} \mathrm{C}$ for $24 \mathrm{~h}$ and the zone of inhibition was determined.

\subsection{Antifungal activity}

The synthesized compounds were screened for the antifungal activity in DMF by poisoned food technique ${ }^{19}$ against Fusarium oxysporum. Prepare Potato Dextrose Agar (PDA) media and about 15 $\mathrm{mL}$ of PDA was poured into each Petri plate and allowed to solidify. $5 \mathrm{~mm}$ disc of seven days old culture of the test fungi was placed at the center of the Petri plate and incubated at $26{ }^{\circ} \mathrm{C}$ for 7 days. After incubation the percentage inhibition was measured and three replicates were maintained for each treatment. Nystatin was used as standard. All the synthesized compounds were tested (at the dosage of $500 \mu \mathrm{L}$ of the new compounds/petriplate, where concentration was $0.1 \mathrm{mg} / \mathrm{mL}$ ) by poisoned food technique.

\section{References}

1 Burger A. (2003) Burger's Medicinal Chemistry and drug Discovery, 6th Ed, John Wiley \& Sons, USA.

2 Sheehan D. J., Hitchcock C. A., and Sibley C. M. (1999) Current and emerging azole antifungal agents. Clin. Microbiol. Rev., 12, 40-79. 
3 Jawad A. H., Shneine J. K., Ahmed A., and Abdulrasool M. M. (2012) Synthesis, characterization and evaluation of biological activity of some heterocyclic compounds containing 1,2,4-triazole ring. Int. J. Research Pharm. Chem., 2, 2231-2781.

4 Omar F. A., Mahfouz N. M., and Rahman M. A. (1996) Synthesis, characterization and crystal structure of ethyl-4-(3-chlorobenzamido)benzoate. Eur. J. Med. Chem., 31, 819-825.

5 Gilani S. J., Khan S. A., and Siddiqui N. (2010) Synthesis and pharmacological evaluation of condensed heterocyclic 6-substituted 1,2,4-triazolo-[3,4-b]-1,3,4-thiadiazole and 1,3,4oxadiazole derivatives of isoniazid. Bioorg. Med. Chem. Lett., 20, 4762-4765.

6 Sahin G., Palaska E., Ekizoglu M., and Ozalp M. (2002) Synthesis and antimicrobial activity of some 1,3,4-oxadiazole derivatives. II Farmaco, 57, 539-542.

7 Wang Z., Wang M., Yao X., Li Y., Qioa W., Geng Y., Liu Y., and Wang Q. (2012) Hydroxyl may not be indispensable for rategravir: Design, synthesis and SAR studies of raltegravir derivatives as HIV-I inhibitors. Eur. J. Med, Chem., 50, 361-369.

8 Bondock S., Adel S., Etman H. A., and Badria F. A. (2012) Synthesis and antitumor evaluation of some 1,3,4-oxadiazoles-based hetrocycles. Eur. J. Med. Chem., 48, 192-199.

9 Bankar G. R., Nandakumar K., Nayak P. G., Thalkur A., Chamallamudi M. R., and Nampurat G. K. (2009) Vasorelaxant effect in rat aortic through calcium channel blockage; Apreliminary in vitro assessment of a 1,3,4-oxadiazole deivatives. Chem. Biol. Interact., 181, 377-382.

10 Liu K., Lu X., Zhang H. J., and Sun, H. L. (2012) Synthesis, molecular and biological evaluation of 2-(benzylthio)-5-aryloxadiazoles derivatives as anti-tumar agents. Eur. J. Med. Chem., 47, 473-478.

11 Gosselin F., Britton R. A., Davies I. W., Dolman S. J., Gauvreau D., Hoerrner R., Hughes G., Janey J., Lau S., and Molinaro C. (2010) A practical synthesis of 5-lipoxygenase inhibitors MK-0633. J. Org. Chem., 75, 4154-4160.

12 Liao Y., Bottcher H., Harting J., Greiner H., Amsterdam C. V., Cremers T., Sundell S., Marz J., Rautenberg W., and Wikstrom H. (2000) New selective and potent 5HT antagonists: Chemistry and pharmacological evaluation of $N$-piperazinylphenyl biphenylcarboxamides and biphenylsulfonamides. J. Med. Chem., 43, 517-525.

13 Chrysina E. D., Kosmopoupou M. N., Tiraidis C., Kardakaris R., Bischler N., Leonidas D. D., Hadady Z., Somsak L., Docsa P., Gergely P., and Oikonomakos N. G. (2005) Kinetic and crystallographic studies on 2-(ß-D-glucopyranosyl)-5-methyl-1,3,4-oxadiazole, benzothiazoleb and -benzimidazole. Protein Sci., 14, 873-888.

14 Huang X. -F., Lu X., Zhang Y., Song G. -Q., He Q. -L., Li Q.-S., Yang X. -H., Wei Y., and Zhu H. -L. (2012) Synthesis, biological evaluation and molecular docking studies of $N$-((1,3diphenyl-1H-pyrazol-4-yl)methyl)aniline derivatives. Bioorg. Med. Chem., 20, 4895-4900.

15 Francisco M., Garibotto, Maximiliano A., Sortino, Vladimir V., Kouznetsov, Ricardo D., Enriz, and Susana A. Z. (2011) Synthesis and antifungal activity of $N$-aryl- $N$-benzylamines and of their homallyl analogues. ARKIVOC, VII, 149-161.

16 Jayashankar B., Lokanath Rai K. M., Baskaran N., and Sathish H. S. (2009) Synthesis and pharmacological evalution of 1,3,4-oxadiazoles bearing bis(hetrocycle) derivatives as antiinflammatory and analgesic agents. Eur. J. Med. Chem., 44, 3898-3902.

17 Om P., Manoj K., Rajesh K., Chetan S., and Aneja K. R. (2010) Hypervalent iodine (III) mediated synthesis of novel unsymmetrical 2,5-disubstituted 1,3,4-oxadiazoles as antibacterial and antifungal agents. Eur. J. Med. Chem., 45, 4252-4257.

18 Andrews J. M. (2001) BSAC standardized disc susceptibility testing method. J. Antimicrob. Chemother., 48, 43-57.

19 Satish S, Mohana D. C., Raghavendra M. P., and Raveesha K. A. (2007) Antifungal activity of some plant extracts against important seed borne pathogens of aspergillus sp. J. Agri. Tech., 3, 109-119. 